\title{
Application of integrated finite differences to compute symmetrical upscaled equivalent conductivity tensor
}

\author{
C. Vassena \& M. Giudici \\ Università degli Studi di Milano, Dip. di Scienze della Terra “A. Desio”, \\ Milano, Italy
}

\begin{abstract}
The equivalent conductivity tensor is computed with a method based on the solution of the balance equation at the fine scale. In particular the balance equation is solved on a block assigning Dirichlet boundary conditions that vary linearly with the space coordinates and the equivalent conductivity tensor is the linear tensor relating average flux and hydraulic gradient. Previous works prove that this method yields a symmetric equivalent conductivity tensor both for continuous domains and for discrete models based on integrated finite differences. Here the equivalent conductivity tensor is computed for two lateral faces of a volume of glacio-fluvial sediments and the results are compared with those obtained with a standard finite differences method on square grids with different spacings.
\end{abstract}

Keywords: upscaling, equivalent conductivity, symmetry, integrated finite differences.

\section{Introduction}

Discrete models of ground water flow are usually based on the discretisation of the subsurface in grid-blocks for which homogeneous equivalent blockscale hydraulic conductivities must be specified. In real porous media the local scale $K$ tensor is heterogeneous within a block and therefore it is necessary to find an equivalent conductivity tensor, $\widetilde{K}$, for each grid-block. The basic idea for upscaling is that the block-averaged Darcy's velocity, $\langle\mathbf{q}\rangle$, and hydraulic gradient, $\langle\mathbf{J}\rangle$, are related by a block-scale Darcy's law: 


$$
\langle\mathbf{q}\rangle=-\widetilde{K}\langle\mathbf{J}\rangle .
$$

Reviews and classifications of the different approaches used to transform a detailed description of the spatial variability of $K$ to a coarser description can be found in $[1,8-10,13,16]$.

The block conductivity tensor is in general non diagonal, because the geometrical regularity of heterogeneity at the fine scale, e.g. layering, yields anisotropy at the coarse scale [14]. Moreover, when eqn. (1) is applied, $\widetilde{K}$ is not an intrinsic property of the block, but depends on the boundary conditions and the source terms. However we can decompose $\langle\mathbf{q}\rangle$ as

$$
\langle\mathbf{q}\rangle=-\widetilde{K}\langle\mathbf{J}\rangle+\mathbf{q}_{n D},
$$

where $\mathbf{q}_{n D}$ represents the non-Darcian block-averaged flow rate. The equivalent conductivity tensor, $\widetilde{K}$, defined by eqn. (1) and computed with the technique proposed in the second section is a local property of the medium at the coarse scale. Non-local effects are described by $\mathbf{q}_{n D}$, which depends on the whole conductivity field, on the boundary conditions, and on the source terms.

Theoretical studies show that the computation of $\widetilde{K}$ with arbitrary boundary conditions might yield a non symmetric tensor, which therefore could not admit principal directions of anisotropy, whereas with some assumptions the symmetry of the block conductivity tensor is demonstrated for instance by $[3,11]$ and [17]. Farmer [4] states sufficient conditions that guarantee the symmetry of $\widetilde{K}$ for a continuous domain: he shows that $\widetilde{K}$ is symmetrical for any conductivity distribution and for any shape of the domain, if it is obtained from eqn. (1) and if $\langle\mathbf{q}\rangle$ is computed by solving forward problems with Dirichlet boundary conditions such that the prescribed head is a linear function of the space coordinates.

In [6] we extend this theorem to a physically based conservative discrete model using the integrated finite differences method (IFD), proposed in the ground water literature by [7] and applied by some authors to model regional flow [5, 12]. In [6] the focus is on 2D stationary flow in a confined aquifer, so that the physical parameter to be considered is the aquifer transmissivity; the proof of the symmetry of the upscaled transmissivity tensor given by [4] is followed and it is rigorously demonstrated that $\widetilde{K}$ is symmetric even in the discrete case for physically based conservative numerical models.

The goal of this paper is to test the result obtained in [6] on some numerical examples: the equivalent $\widetilde{K}$ tensor is computed for two faces of a volume of glaciofluvial sediments belonging to Pleistocene sequences of the Ticino basin (Northern Italy) described in [15]. We show that IFD can be useful to model media which are the union of many zones, each of which characterized by a single hydrofacies: in fact this method permits the discretisation of the discontinuities between different zones in a more accurate way than standard finite differences techniques which cannot handle non-rectangular grid cells. 


\section{The computation of the symmetric equivalent conductivity tensor}

We refer to discrete models for which the porous medium under study is subdivided into coarse blocks, and each coarse block is subdivided into a fine grid of cells, so that at each fine cell the conductivity takes a different value. For the sake of simplicity we refer to 2D flow in the vertical $x-z$ plane, but the basic results can be easily extended to 3D flow.

The block conductivity tensor is computed with a local-numerical approach [9], or local-local technique according to the classification of [4]. In the local methods the equivalent block conductivity is assumed to depend only on the local $K$ values inside the block. Numerical techniques are based on the numerical solution of balance equations: the spatially averaged flow through each block is computed from the solution of the flow problems at the fine scale and eqn. (1) permits to compute an upscaled conductivity tensor for the coarse scale blocks.

Virtual experiments are conducted on a block, solving the discrete balance equation on the fine grid; the boundary conditions are assigned at the border of each block as prescribed heads that are linear functions of the space coordinates.

The fine grid is based on a set of nodes, around which Voronoi polygons (fig. 1) are built as the union of the mediators of each segment joining adjacent nodes [2, p. 353]. Each node will be denoted either by an integer index, e.g. $j$, or by its position vector, e.g. $\mathbf{x}_{j}=\left(\left(x_{1}\right)_{j},\left(x_{2}\right)_{j}\right)$; the node index is used to label the cell corresponding to the domain enclosed by a Voronoi polygon.

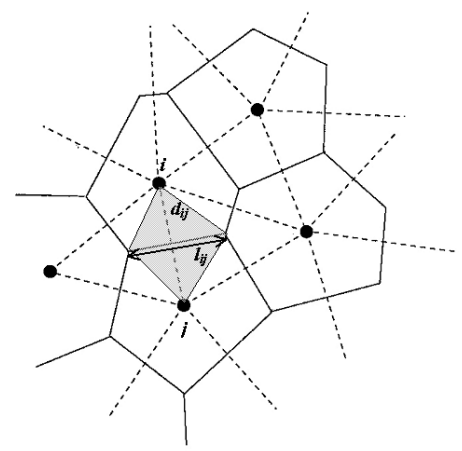

Figure 1: Grid built with Voronoi polygons. $d_{i, j}$ is the length of the internode segment connecting nodes $i$ and $j ; l_{i, j}$ is the length of the side separating the cells $i$ and $j$; the area of the gray region is $\left(d_{i, j} l_{i, j}\right) / 2$ (from [6]).

Let $N$ be the set of nodes which belong to a coarse block, and let $N=N_{0} \cup N_{b}$, where $N_{0}$ is the subset of nodes for which the balance equation can be written, $N_{b}$ is the subset of the boundary nodes. 
The balance equation for any cell $j \in N_{0}$, assuming that the source terms are null, has the form:

$$
\frac{1}{A_{j}} \sum_{i \in S_{j}} K_{i, j} \frac{h_{i}-h_{j}}{d_{i, j}} l_{i, j}=0
$$

where: $S_{j}$ is the subset of nodes connected to the $j$-th node; $A_{j}$ is the area of the $j$-th cell; $K_{i, j}$ is the internode conductivity; $h_{j}$ is the piezometric head at the $j$-th node; $d_{i, j}=\left\|\mathbf{x}_{i}-\mathbf{x}_{j}\right\|$ is the distance between the $i$-th and $j$-th nodes; $l_{i, j}$ is the length of the side separating the $i$-th and $j$-th cells.

Each of the terms $K_{i, j}\left(h_{i}-h_{j}\right) d_{i, j}^{-1} l_{i, j}$ appearing in the summation of eqn. (3) corresponds to the flux per unit length along $y$ direction entering in the $j$-th cell through the side separating it from the $i$-th cell.

Notice that the standard finite differences method can be viewed as a special case of the IFD method, in particular for square cells $l_{i, j}=d_{i, j}=\Delta x$, where $\Delta x$ is the grid spacing.

Let the boundary conditions on the piezometric head assigned at the border of the coarse block be linear:

$$
h_{i}^{(r)}=\mathbf{x}_{i} \cdot \mathbf{e}^{(r)}, i \in N_{b}
$$

where

$$
\mathbf{e}^{(1)}=(1,0), \mathbf{e}^{(2)}=(0,1)
$$

and the index $r$ refers to the $r$-th virtual experiment; the piezometric head $h^{(r)}$ satisfies eqn. (3).

In the numerical tests the equivalent tensor $\widetilde{K}$ is computed from eqn. (1) and is given by:

$$
\widetilde{K}_{s r}=\frac{1}{A} \sum_{j \in N} \sum_{\substack{i \in S_{j} \\ i \leq j}} K_{i, j} \frac{h_{i}^{(r)}-h_{j}^{(r)}}{d_{i, j}} \frac{l_{i, j} d_{i, j}}{2} \frac{\left(x_{s}\right)_{i}-\left(x_{s}\right)_{j}}{d_{i, j}}
$$

where

$$
A=\sum_{j \in N} \sum_{\substack{i \in S_{j} \\ i \leq j}} \frac{l_{i, j} d_{i, j}}{2} .
$$

In [6] the same track of the proof given by [4] for the continuous case is followed; gradients are substituted with finite differences approximations and integrals are substituted with sums over a block.

The following expression, equivalent to eqn. (5), is obtained for the components of $\widetilde{K}$ and implies the symmetry of the equivalent conductivity tensor at the coarse scale:

$$
\widetilde{K}_{s r}=\frac{1}{A} \sum_{j \in N} \sum_{\substack{i \in S_{j} \\ i \leq j}} K_{i, j} \frac{h_{i}^{(r)}-h_{j}^{(r)}}{d_{i, j}} \frac{h_{i}^{(s)}-h_{j}^{(s)}}{d_{i, j}} \frac{l_{i, j} d_{i, j}}{2} .
$$

The index $s$ refers to the direction of flow, whereas $r$ refers to the component of the hydraulic gradient. Notice that $l_{i, j} d_{i, j} / 2$ is the surface of the gray area in fig. 1 . 
An alternative way to compute $\widetilde{K}[6]$ is based on the equivalence between the dissipated energy averaged over a block, $-\langle\mathbf{q} \cdot \mathbf{J}\rangle$, and computed from block averaged quantities, $-\langle\mathbf{q}\rangle \cdot\langle\mathbf{J}\rangle$, i.e. the equivalent conductivity tensor is obtained with the condition that

$$
-\langle\mathbf{q} \cdot \mathbf{J}\rangle=-\langle\mathbf{q}\rangle \cdot\langle\mathbf{J}\rangle=\sum_{m=1}^{2} \sum_{n=1}^{2} \widetilde{K}_{m, n}\left\langle J_{m}\right\rangle\left\langle J_{n}\right\rangle .
$$

In [6] we show that the block conductivity tensors computed from the criteria of equivalence of flux and of energy dissipation coincide if fixed head boundary conditions that vary linearly in space are assigned.

\section{A case study}

The local-numerical approach described in section 2 is applied to Pleistocene sequences of the Ticino basin (northern Italy), where some volumes of glaciofluvial sediments outcropping at a quarry site are investigated at the meter scale [15]. Here we consider two lateral faces (labelled by A and C in [15]) of one of these volumes. In [15] a simplified scheme of "operative facies" is obtained by grouping facies into five categories, each characterised by a constant conductivity value, obtained from laboratory tests or estimated with empirical formulas. The boundaries between individual facies and depositional units are drawn in fig. 2, where different operative facies are represented with different shadings.

The flow model is applied to each face under the assumption of 2D flow, and $\widetilde{K}$ is computed at the block scale, considering the whole face as a block.

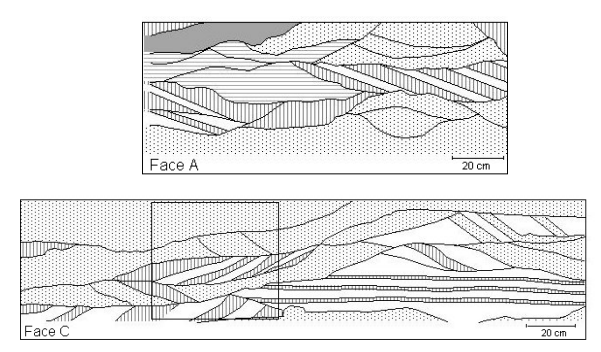

Figure 2: Sedimentological interpretation and operative facies of Faces A and C. Grey: open framework gravel $\left(K=5 \cdot 10^{-2} \mathrm{~m} / \mathrm{s}\right)$; horizontal lines: sandy gravel well sorted $\left(K=2 \cdot 10^{-3} \mathrm{~m} / \mathrm{s}\right)$; dots: sandy gravel poorly sorted $\left(K=6 \cdot 10^{-4} \mathrm{~m} / \mathrm{s}\right)$; vertical lines: coarse to medium sand $\left(K=5 \cdot 10^{-4} \mathrm{~m} / \mathrm{s}\right)$; white: fine sand $\left(K=10^{-4} \mathrm{~m} / \mathrm{s}\right)$.

Notice that in [15] cobbles with diameter greater than $2 \mathrm{~cm}$ are considered as impermeable bodies to perform the 3D flow modeling; here we do not consider those features, because their use in a 2D flow model introduces impermeable 
structures with a large and non realistic lateral extension along the direction orthogonal to the face.

The tensor $\widetilde{K}$ is computed using the local-numerical approach based on the solution of the balance equation with the IFD method: for each face two examples of Voronoi grids are considered and the results are then compared with those obtained with a standard finite differences technique on square grids with different spacings. The Voronoi grids are a coarse one (IC), with nodes placed only along the discontinuities among operative facies, and a fine one (IF) obtained by adding some nodes far from the discontinuities.

Table 1: Equivalent conductivity tensors $\left(10^{-4} \mathrm{~m} / \mathrm{s}\right) . \mathrm{R} n$ indicates the regular grid with spacing of $n \mathrm{~cm}$; IC indicates the results obtained with the coarse irregular grid, IF those obtained with the fine grid.

Face A

\begin{tabular}{|c|c|c|c|c|c|c|c|}
\hline Grid & $\mathrm{R} 8$ & $\mathrm{R} 4$ & $\mathrm{R} 2$ & $\mathrm{R} 1$ & $\mathrm{R} 0.5$ & $\mathrm{IC}$ & $\mathrm{IF}$ \\
\hline$\widetilde{K}_{x x}$ & 17 & 26 & 29 & 30 & 31 & 32 & 32 \\
$\widetilde{K}_{x z}$ & 3.9 & 3.4 & 4.3 & 4.0 & 3.9 & 4.2 & 4.2 \\
$\widetilde{K}_{z x}$ & 4.4 & 3.6 & 4.5 & 4.1 & 3.9 & 4.2 & 4.2 \\
$\widetilde{K}_{z z}$ & 5.4 & 5.2 & 5.8 & 5.8 & 5.8 & 6.3 & 6.2 \\
\hline
\end{tabular}

Face C

\begin{tabular}{|c|c|c|c|c|c|c|c|}
\hline Grid & R8 & R4 & R2 & R1 & R0.5 & IC & IF \\
\hline$\widetilde{K}_{x x}$ & 4.0 & 4.1 & 4.0 & 4.1 & 4.1 & 4.1 & 4.1 \\
$\widetilde{K}_{x z}$ & 0.15 & 0.06 & 0.11 & 0.10 & 0.10 & 0.087 & 0.095 \\
$\widetilde{K}_{z x}$ & 0.18 & 0.10 & 0.12 & 0.11 & 0.10 & 0.094 & 0.099 \\
$\widetilde{K}_{z z}$ & 3.7 & 3.4 & 3.1 & 3.1 & 3.1 & 3.0 & 3.0 \\
\hline
\end{tabular}

In table 1 the components of $\widetilde{K}$ computed with different grids are listed.

The dominant components are the diagonal ones, especially for face $\mathrm{C}$, for which the off-diagonal terms are less than the diagonal values by more than one order of magnitude: we can conclude that $x$ and $z$-axis can be considered as the principal axes of $\widetilde{K}$.

The off-diagonal terms, $\widetilde{K}_{x z}$ and $\widetilde{K}_{z x}$, are computed with eqn. (5) and show differences which are less than $20 \%$ of the computed values for the coarsest grids (R8), but become negligible for fine grids and for irregular grids. The difference $\widetilde{K}_{x z}-\widetilde{K}_{z x}$ is due to the approximations introduced in the solution of the balance equation and in the computation of $\widetilde{K}$ with eqn. (5), which does not explicitly imply symmetry, and is an estimate of the uncertainties on the components of $\widetilde{K}$.

Table 1 also permits to draw some conclusions about the dependence of the results on the grid spacings. For these tests the results obtained with the IFD 
method do not noticeably depend upon the refinement of the grid: both grids (IC and IF) accurately reproduce the discontinuities between the facies, which is the most important aspect; moreover, the differences between the results obtained with IC and IF are small if compared with the differences between the sizes of the cells of the grids (table 2). The surface of the cells of the regular grids varies between $64 \mathrm{~cm}^{2}$ (R8) and $0.25 \mathrm{~cm}^{2}$ (R0.5) and, as noticed before, the results are influenced by the the size of the cells.

A direct comparison between the IC and IF is given in fig. 3, where a small area of face $\mathrm{C}$ is represented as an example, whereas a more complete analysis of the characteristics of the irregular grids can be found in table 2 .

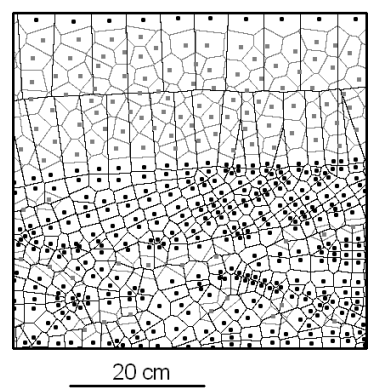

Figure 3: Comparison between the irregular grids IC (black) and IF (gray) in the small area belonging to face $\mathrm{C}$ indicated by a square in fig. 2 .

Fig. 3 shows that the coarse grid reproduces in a sufficiently accurate way the boundary of the discontinuities but it is constituted by cells of irregular shapes and noticeably different size from each other.

Quantitative information about the shape of the cells is given by a shape factor, defined as $\sigma_{j}=l_{j} / r_{j}$, where $l_{j}$ is the diameter of the $j$-th cell and $r_{j}$ is the radius of the inscribed circle. We have $\sigma=2$ if the cell is round, $\sigma=2 \sqrt{2}$ for a square cell, whereas higher values of $\sigma$ characterize a cell with a more irregular shape.

In table 2 the maximum and the average values of the shape factor are given for each irregular grid: in particular the values of the maximum and the standard deviation are useful to assess the enhanced regularity of the fine grids with respect to the coarse ones and the wide range of different shapes and sizes of the Voronoi cells in comparison with the regular ones.

\section{Conclusions}

In [6] the following theorem is proven. Let the discrete balance equation (3) be valid over a Voronoi diagram, with which a block is discretized to apply an IFD model. Let $h^{(r)}$ be the solution to (3) if Dirichlet boundary conditions are assigned so that the prescribed head is a linear function of $x_{r}$. The block 
Table 2: Parameters of the irregular grids for both faces. In the last column average and standard deviation of the shape factor $\sigma$ are listed.

\begin{tabular}{|c|c|c|c|c|}
\hline Grid & Number of nodes & $\max _{j \in N_{0}}\left(A_{j}\right)\left(\mathrm{cm}^{2}\right)$ & $\max _{j \in N_{0}}\left(\sigma_{j}\right)$ & $\sigma$ \\
\hline IC-A & 503 & 68 & 34.1 & $5.8 \pm 4.1$ \\
IF-A & 752 & 35 & 18.1 & $3.8 \pm 2.1$ \\
IC-C & 1157 & 127 & 40.4 & $6.0 \pm 4.2$ \\
IF-C & 1685 & 18 & 12.6 & $4.1 \pm 1.7$ \\
\hline
\end{tabular}

scale equivalent conductivity tensor computed from (1) is symmetric for any conductivity distribution and for any shape of the block.

A local-numerical approach is adopted: the discrete balance equation is solved on the fine grid with boundary conditions assigned at the border of each block and the equivalent conductivity tensor is computed as the coefficient of proportionality between the block averaged Darcy's velocity and the block averaged hydraulic gradient.

This result is obtained in [6] for the IFD method that is based on a balance equation and permits to approximate the discontinuities between different zones in a more accurate way than standard finite differences techniques.

The numerical tests show that, as expected from the theory, the equivalent conductivity tensors that control the Darcian term of the block-averaged flux are symmetric but for differences between off-diagonal terms due to approximation and rounding errors in the computation of $\widetilde{K}$.

Moreover, the IFD method is useful to model media which are the union of many hydrofacies and permits the discretisation of the discontinuities between different zones in a more accurate way than standard finite differences techniques which cannot handle non-rectangular grid cells.

\section{Acknowledgements}

This work has been supported by the Italian Ministry for University and Scientific Research (PRIN 2005) and the University of Milan within the project "Field and numerical studies to model the sedimentary architecture and water flow in aquifer systems of the Po plain at different scales" (principal investigator: M. Giudici).

\section{References}

[1] Cushman, J.H., Bennethum, L.S. \& Hu, B.X., A primer on upscaling tools for porous media. Adv. Water Res., 25(8-12), pp. 1043-1067, 2002.

[2] de Marsily, G., Quantitative Hydrogeology, Academic Press: Orlando, pp. $440,1986$. 
[3] Durlofsky, L.J., Numerical calculation of equivalent grid block permeability tensors for heterogeneous porous media. Water Resources Res., 27(5), pp. 699-708, 1991.

[4] Farmer, C.L., Upscaling: a review. Int. J. Numer. Meth. Fluids, 40(1-2), pp. 63-78, 2002.

[5] Fogg, G.E., Groundwater flow and sand body interconnectedness in a thick, multiple-aquifer system. Water Resources Res., 22(5), pp. 679-694, 1986.

[6] Giudici, M. \& Vassena, C., About the symmetry of the upscaled equivalent transmissivity tensor. Math. Geol., accepted, 2007.

[7] Narasimhan, T. \& Witherspoon, P.A., An integrated finite-difference method for analyzing fluid flow in porous media. Water Resources Res., 12(1), pp. 57 64, 1976.

[8] Nœtinger, B., Artus, V. \& Zargar, G., The future of stochastic and upscaling methods in hydrogeology. Hydrogeol. J., 13, pp. 184-201, 2005.

[9] Renard, Ph. \& de Marsily, G., Calculating equivalent permeability: a review. Adv. Water Res., 20(5-6), pp. 253-278, 1997.

[10] Sanchez-Vila, X., Guadagnini, A. \& Carrera, J., Representative hydraulic conductivities in saturated groundwater flow. Rev. Geophys., 44, doi:10.1029/2005RG000169, 2006.

[11] Trykozko, A., Zijl, W. \& Bossavit, A., Nodal and mixed finite elements for the numerical homogenization of 3D permeability. Comput. Geosci., 5, pp. 61-84, 2001.

[12] Vassena, C., Sviluppo e applicazione di modelli di flusso delle acque sotterranee nella conoide alluvionale del fiume Reno, $\mathrm{PhD}$ thesis, University of Milan, Italy, pp. 147, 2004.

[13] Wen, X.-H. \& Gomez-Hernandez, J.J., Upscaling hydraulic conductivity in heterogeneous media: an overview. Jour. Hydrology, 183(1-2), pp. ix-xxxii, 1996.

[14] White, C.D. \& Horne, R.N., Computing absolute transmissivity in the presence of fine scale heterogeneity. Society of Petroleum Engineers, SPE 16011, pp. 209-221, 1987.

[15] Zappa, G., Bersezio, R., Felletti, F., \& Giudici, M., Modeling heterogeneity of gravel-sand, braided stream, alluvial aquifers at the facies scale. Jour. Hydrology, (325), pp. 134-153, 2006.

[16] Zijl, W., Scale aspects of groundwater flow and transport systems. Hydrogeol. J., (7), pp. 139-150, 1999.

[17] Zijl, W. \& Trykozko, A., Numerical homogeneization of the absolute permeability using the conformal-nodal and mixed-hybrid finite element method. Transp. Porous Media, (44), pp. 33-62, 2001. 